\title{
Outcomes of cardiac surgery in chronic kidney disease
}

\author{
Mangalee Fernando, FRACP, ${ }^{\mathrm{a}, \mathrm{b}}$ Hugh S. Paterson, FRACS, ${ }^{\mathrm{c}}$ Karen Byth, PhD, ${ }^{\mathrm{d}}$ \\ Benjamin M. Robinson, MBBS, MPhil, ${ }^{\mathrm{e}}$ Hugh Wolfenden, FRACS, ${ }^{\mathrm{f}}$ David Gracey, PhD, FRACP, ${ }^{\mathrm{c}, \mathrm{g}}$ and \\ David Harris, MD, BS, FRACP ${ }^{\mathrm{c}, \mathrm{h}}$
}

Objective: To identify predictors of early and late outcomes of cardiac surgery in patients with chronic kidney disease.

\begin{abstract}
Methods: Patients $(\mathrm{n}=545)$ with serum creatinine $\geq 200 \mu \mathrm{mol} / \mathrm{L}$ or renal dialysis were identified from databases maintained by the largest Sydney cardiothoracic surgical units with data consistent with the Australian and New Zealand Society of Cardiothoracic Surgeons data definitions. The patient data were matched against the National Dialysis Database and the New South Wales Register of Births, Deaths, and Marriages. Statistical analysis was used to identify predictors of early and late outcomes.
\end{abstract}

\begin{abstract}
Results: The Kaplan-Meier estimate of 1-, 5-, and 10-year survival for all patients was $78 \%, 56 \%$, and 36\%, respectively. The outcomes were similar after coronary bypass surgery and valve replacement and were also similar for dialysis and nondialysis patients. The odds ratios for the significant independent predictors of outcomes were, for perioperative death, age (1.4 per decade), emergency surgery (7.0), redo surgery (3.8), left ventricular impairment (moderate, 2.7; severe, 4.4); for new early postoperative dialysis, estimated glomerular filtration rate $<20 \mathrm{~mL} / \mathrm{min}$ (3.8), emergency surgery (2.7), tricuspid valve surgery (4.4); for new permanent dialysis within 6 months of surgery, serum estimated glomerular filtration rate $<20 \mathrm{~mL} / \mathrm{min}$ (odds ratio, 4.6). The hazard ratio for the independent predictors of late death in those alive 6 months after surgery was 1.4 per decade for age and 1.4 for moderate or severe left ventricular impairment.
\end{abstract}

Conclusions: Left ventricular impairment is a risk factor for perioperative and late death in patients with kidney disease. After cardiac surgery, preoperative dialysis-dependent and dialysis-free patients had similar long-term outcomes. (J Thorac Cardiovasc Surg 2014;148:2167-73)

Chronic kidney disease (CKD) has consistently been found to be an independent risk factor for the development of cardiovascular disease. ${ }^{1,2}$ The leading cause of death in patients with CKD is cardiovascular disease. Several studies have identified that CKD increases the risk of mortality in patients undergoing cardiac surgery and that the mortality increases with renal dysfunction severity. ${ }^{3,4}$ However, the heterogeneity among these patients will be wide, because the comorbidities, such as diabetes, baseline cardiac function, and symptoms, will vary. Providing individual patients with specific prognostic data

\footnotetext{
From the Departments of Nephrology ${ }^{\mathrm{a}}$ and Cardiothoracic Surgery, ${ }^{\mathrm{f}}$ Prince of Wales Hospital, Sydney, Australia; University of New South Wales, ${ }^{b}$ Sydney, Australia; University of Sydney, ${ }^{\mathrm{c}}$ Sydney, Australia; Westmead Hospital and University of Sydney, ${ }^{\mathrm{d}}$ Sydney, Australia; Departments of Cardiothoracic Surgery ${ }^{\mathrm{e}}$ and Nephrology, ${ }^{\mathrm{g}}$ Royal Prince Alfred Hospital, Sydney, Australia; and Department of Nephrology, ${ }^{\text {h }}$ Westmead Hospital, Sydney, Australia.

Disclosures: Authors have nothing to disclose with regard to commercial support.

Drs Fernando and Paterson contributed equally to this work.

Received for publication Aug 9, 2013; revisions received Nov 24, 2013; accepted for publication Dec 10, 2013; available ahead of print Feb 9, 2014.

Address for reprints: Mangalee Fernando, FRACP, Department of Nephrology, Prince of Wales Hospital, Level 3 High St, Building Barker St, Randwick, NSW 2031, Australia (E-mail: m.fernando@unsw.edu.au). $0022-5223 / \$ 36.00$

Crown Copyright $\odot 2014$ Published by Elsevier Inc. on behalf of The American Association for Thoracic Surgery

http://dx.doi.org/10.1016/j.jtcvs.2013.12.064
}

and evaluating their suitability for surgery is often a challenge. Patients with advanced kidney disease often request information on whether the surgery will precipitate a requirement for permanent dialysis. In the present study, we aimed to identify the predictors of perioperative and postdischarge mortality in patients with dialysis-dependent (DD) or nondialysis-dependent (NDD) CKD. We also aimed to identify the factors that would predict long-term dialysis in predialysis patients who were discharged after cardiac surgery.

\section{METHODS}

In the present multicenter study, we compared and linked the existing cardiothoracic databases with the New South Wales Register of Births, Deaths, and Marriages and with corresponding renal databases in 3 teaching hospitals based in Sydney, Australia. The patients with a last preoperative serum creatinine measurement $\geq 200 \mu \mathrm{mol} / \mathrm{L}$ or requiring preoperative dialysis were identified from the cardiac surgical databases in 3 of the larger cardiothoracic surgical departments in Sydney. The patients with functioning renal transplants were excluded. The estimated glomerular filtration rate (eGFR) was calculated from the Modification of Diet in Renal Disease formula, where eGFR $\mathrm{mL} / \mathrm{min}=32,788 \times(\text { serum creatinine } \mu \mathrm{mol} / \mathrm{L})^{-1.154} \times($ age in years $)^{-0.203} \times(0.742$ if female $)$. The formula does not account for the body surface area and is based on an eGFR of $\mathrm{mL} / \mathrm{min} / 1.73 \mathrm{~m}^{2}$ body surface area. The patients were classified as preoperatively DD or preoperatively NDD. The periods of data collection were January 1996 to June 2010 for Prince of Wales Hospital (POWH), September 2005 to December 2010 for Royal Prince Alfred Hospital (RPAH), and September 


$$
\begin{aligned}
& \text { Abbreviations and Acronyms } \\
& \begin{aligned}
\text { CAG } & =\text { coronary artery graft surgery } \\
\text { CKD } & =\text { chronic kidney disease } \\
\text { DD } & =\text { dialysis dependent } \\
\text { LVEF } & =\text { left ventricular ejection fraction } \\
\text { NDD } & =\text { nondialysis dependent } \\
\text { OR } & =\text { odds ratio } \\
\text { POWH } & =\text { Prince of Wales Hospital } \\
\text { RPAH } & =\text { Royal Prince Alfred Hospital }
\end{aligned}
\end{aligned}
$$

1999 to March 2009 for Westmead Hospital (WH). The human research ethics committee of each hospital approved the present study.

\section{Surgical Data Acquisition}

The data were obtained from cardiothoracic surgical databases in the 3 participating hospitals. All 3 cardiothoracic surgical units collected data, in keeping with the Australian and New Zealand Society of Cardiothoracic Surgeons data definitions. Follow-up data were obtained by matching the names against the hospital dialysis databases and against the New South Wales Register of Births, Deaths, and Marriages. A total of 39 operative and preoperative variables (with major variables listed in Table 1) were analyzed in relation to the following outcomes in the specified patient groups:

1. Perioperative death (during surgery or same admission, all patients)

2. Early postoperative dialysis (NDD patients)

3. New permanent dialysis (within 6 months of surgery for discharged NDD patients)

4. Interval to death for those surviving 6 months after surgery ("late death landmark analysis")

\section{Long-Term Renal and Mortality Data}

The nephrology departments of all 3 hospitals maintained data collected prospectively from dialysis and transplantation patients and provided yearly input to the national data base (Australian and New Zealand Dialysis and Transplant Registry). In the few patients for whom no data were available, the hospital medical records were used. The data were also matched with the New South Wales Registry of Births, Deaths, and Marriages, which is linked to the death registers in the other Australian states. The follow-up of patients through the hospital databases and the New South Wales Register of Births, Deaths, and Marriages has demonstrated a better than $99 \%$ accuracy. ${ }^{5}$

\section{Statistical Analysis}

The statistical software programs, Statistical Package for Social Sciences, version 21, and S-PLUS, version 8, were used to analyze the data. Two-tailed tests with a significance level of $5 \%$ were used throughout. The chi-square or Fisher's exact test, as appropriate, were used to test for an association between the categorical variables. The 2-sample $t$ tests were used to test for differences in the age distribution. Kaplan-Meier survival curves were used to illustrate the survival distributions, and log-rank tests were used to test for differences among the subgroups. The 1-, 5-, and 10-year overall survival rates and their $95 \%$ confidence intervals ([CI], Greenwood formula) were calculated.

Variables with a univariate association of $P<.1$ were considered candidates for inclusion in multiple logistic regression models for each of the dichotomous outcomes (perioperative death, dialysis in the postoperative period for NDD patients, and new permanent dialysis within
6 months of surgery for discharged NDD patients). The eGFR calculated from the preoperative serum creatinine was separated into 3 groups of NDD patients $(<20,20-25$, and $>25 \mathrm{~mL} / \mathrm{min})$ comprising approximately equal numbers in each group. When statistical significance for eGFR has been expressed, it was relative to the group with eGFR $>25 \mathrm{~mL} / \mathrm{min}$. All analyses reported were from the use of eGFR values and groups. The preoperative serum creatinine was also used to define 3 groups (200-250, 251-350, and $>350 \mu \mathrm{mol} / \mathrm{L}$ ), and the analyses were repeated, substituting the serum creatinine values and groups for the eGFR values and groups. When a significance for creatinine has been expressed, it was relative to the group with preoperative serum creatinine 200 to $250 \mu \mathrm{mol} / \mathrm{L}$. Backward stepwise variable selection was used to identify the independent predictors of each dichotomous outcome. Odds ratios (ORs) and their $95 \%$ CIs were used to quantify the strength of the association.

For patients alive 6 months after surgery, a landmark analysis was used to assess the effect of permanent dialysis status at 6 months on late survival and to identify the independent predictors of the interval to "late death." Variables exhibiting $P<.1$ on univariate analysis were considered candidates for inclusion in multiple Cox regression models. Backward stepwise variable selection was used to identify the independent predictors. The hazard ratios and their $95 \%$ CIs were used to quantify the strength of association.

\section{RESULTS \\ Patient Characteristics}

A total of 15,816 patients (POWH, 7583; RPAH, 2670; WH, 5563) underwent cardiothoracic surgery in the 3 surgical units during the periods of data collection, with 545 meeting the criteria for preoperative CKD. Of the 545 patients with preoperative CKD, 196 (36\%) were DD and $349(64 \%)$ were NDD. Two thirds were male, with a mean age of $65.5 \pm 12.4$ years, and the median follow-up period was 8.4 years $(95 \%$ CI, 7.3-9.5). The major patient characteristics and significant differences between the DD and NDD patients and between the CKD and other cardiac surgery patients are listed in Table 1.

\section{Overall Survival}

The data in Figure 1 shows that the 1-, 5-, and 10-year survival estimate for all patients with CKD was $78 \%$, $56 \%$, and $36 \%$, respectively, with no significant difference in overall survival between the DD and NDD groups $(P=.615)$. The 1-, 5-, and 10-year survival estimates with $95 \%$ CIs for the various subgroups of patients are listed in Table 2.

\section{Perioperative Death}

The overall perioperative mortality was 76 of 545 $(13.9 \%)$, and all 3 hospitals had similar rates (POWH, $14.3 \%$; WH, $13.8 \%$; RPAH, $13.5 \%$ ). Of the 76 who died, 47 were men and 29 were women $(P=.063) ; 57(16.3 \%)$ were NDD patients and $19(9.7 \%)$ were DD patients $(P=.032)$. The perioperative mortality for primary, isolated, nonemergency coronary artery graft surgery (CAG) was $5.8 \%$, and for isolated CAG, it was $9.4 \%$. Multiple logistic regression analysis of all patients with 
TABLE 1. Major preoperative and operative variables for NDD, DD, and other cardiac surgical groups

\begin{tabular}{|c|c|c|c|c|c|c|}
\hline \multirow[b]{2}{*}{ Variable } & \multicolumn{4}{|c|}{ Cardiac surgery patients with renal failure } & \multirow[b]{2}{*}{$\begin{array}{c}\text { Other cardiac surgery } \\
\text { patients }(n=15,271)(\%)\end{array}$} & \multirow[b]{2}{*}{$P$ value } \\
\hline & $\begin{array}{c}\text { Creatinine } \geq 200 \\
(\mathrm{NDD}, \mathbf{n}=\mathbf{3 4 9})(\%)\end{array}$ & $\begin{array}{c}\text { Dialysis } \\
(\mathrm{DD}, \mathbf{n}=196)(\%)\end{array}$ & $P$ value* & $\begin{array}{l}\text { CKD subtotal } \\
(n=545)(\%)\end{array}$ & & \\
\hline \multicolumn{7}{|l|}{ Preoperative } \\
\hline Age (y) & $67.5 \pm 12.1$ & $61.8 \pm 12.1$ & $<.001$ & $65.5 \pm 12.4$ & $62.3 \pm 12.1$ & $<.001$ \\
\hline Female gender & 29 & 30 & .721 & 29 & 28 & .57 \\
\hline Symptom class IV & 32 & 26 & .199 & 30 & 21 & $<.001$ \\
\hline Diabetes & 35 & 38 & .558 & 36 & 27 & $<.001$ \\
\hline $\mathrm{LVEF}<30 \%$ & 13 & 7 & .047 & 11 & 4.3 & $<.001$ \\
\hline \multicolumn{7}{|l|}{ Surgery } \\
\hline Elective & 34 & 37 & .146 & 35 & 56 & $<.001$ \\
\hline Urgent & 52 & 54 & & 53 & 40 & \\
\hline Emergency & 14 & 9 & & 12 & 4.3 & \\
\hline Peripheral vascular disease & 21 & 19 & .623 & 20 & 10 & $<.001$ \\
\hline Airways disease & 14 & 11 & .349 & 13 & 12 & .43 \\
\hline \multicolumn{7}{|l|}{ Operative } \\
\hline Total CAG $+\ddagger$ & 75 & 75 & .894 & 74 & 78 & .026 \\
\hline Isolated CAG & 59 & 57 & .717 & 58 & 67 & $<.001$ \\
\hline Valve replacement & 33 & 34 & .718 & 33 & 20 & $<.001$ \\
\hline Multiple procedure & 22 & 26 & .361 & 23 & 18 & .004 \\
\hline Redo cardiac surgery & 16 & 9 & .015 & 13 & 5.2 & $<.001$ \\
\hline
\end{tabular}

$N D D$, Nondialysis dependent; $D D$, dialysis dependent; $C K D$, chronic kidney disease; $L V E F$, left ventricular ejection fraction; $C A G$, coronary artery graft surgery. ${ }^{*}$ NDD versus DD patients. $\lceil$ CKD versus other cardiac surgery patients. $₫$ CAG with other cardiac procedures.

CKD identified emergency surgery (OR, 6.3; 95\% CI, 3.0-13.6; $P<.001)$, preoperative left ventricular ejection fraction (LVEF) $(30 \%-50 \%$ : OR, 2.8; 95\% CI, 1.5-5.2; $P=.001$; and $<30 \%$ : OR, 4.3; 95\% CI, 2.0-9.1; $P<.001$ ), age (OR, 1.4 per decade; 95\% CI, 1.1-1.8; $P=.008)$, redo surgery (OR, 4.0; 95\% CI, 2.1-7.7; $P<.001)$, and female gender (OR, 2.3; 95\% CI, 1.3-4.1;
$P=.004)$ as independent predictors of perioperative mortality. After adjusting for these independent predictors, no significant association was found between perioperative mortality and preoperative dialysis status (DD vs NDD, $P=.724)$ nor with chronic lung disease, preoperative creatinine, preoperative angina status, peripheral vascular disease, or operative time.
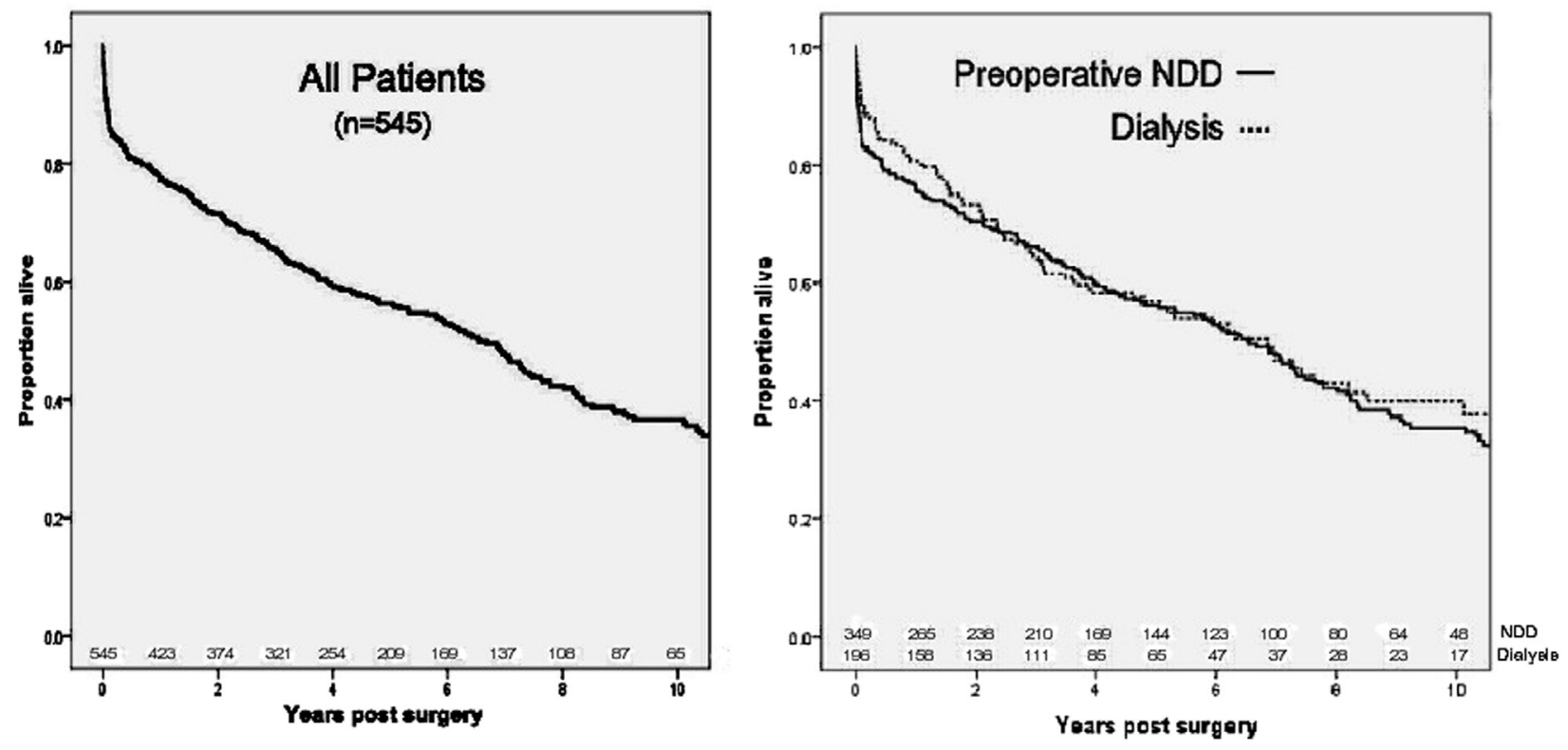

FIGURE 1. Kaplan-Meier curves of overall survival for all patients with chronic kidney disease and stratified by preoperative dialysis status. $N D D$, Nondialysis dependent. 
TABLE 2. Kaplan-Meier estimates of survival stratified by category

\begin{tabular}{|c|c|c|c|c|c|c|}
\hline \multirow{2}{*}{ Variable } & \multicolumn{2}{|c|}{ 1-y Survival } & \multicolumn{2}{|c|}{ 5-y Survival } & \multicolumn{2}{|c|}{ 10-y Survival } \\
\hline & $\%$ & $95 \% \mathbf{C I}$ & $\%$ & $95 \% \mathrm{CI}$ & $\%$ & $95 \% \mathbf{C I}$ \\
\hline \multicolumn{7}{|l|}{ All patients $(\mathrm{n}=545)$} \\
\hline All patients & 78 & $74-81$ & 56 & $52-60$ & 36 & $31-41$ \\
\hline Preoperative DD & 81 & $75-86$ & 56 & $49-63$ & 39 & $30-48$ \\
\hline Preoperative NDD & 76 & $71-80$ & 56 & $51-62$ & 35 & $29-41$ \\
\hline CAG only & 83 & $79-87$ & 59 & $54-65$ & 37 & $29-42$ \\
\hline Valve replacement surgery & 72 & $66-79$ & 54 & $46-62$ & 38 & $30-48$ \\
\hline \multicolumn{7}{|c|}{ Survival from 6-mo landmark $(\mathrm{n}=441)$} \\
\hline Late dialysis & 95 & $92-98$ & 66 & $59-73$ & 44 & $34-54$ \\
\hline No late dialysis & 97 & 94-99 & 72 & $66-77$ & 46 & $38-54$ \\
\hline CAG only, dialysis & 97 & $93-100$ & 73 & $63-81$ & 42 & $28-56$ \\
\hline Valve, dialysis & 93 & $87-100$ & 62 & $48-75$ & 50 & $35-65$ \\
\hline
\end{tabular}

$C I$, Confidence interval; $D D$, dialysis dependent; $N D D$, nondialysis dependent; $C A G$, coronary artery graft surgery.

\section{Early Postoperative Dialysis}

Of the 349 NDD patients, $54(15.5 \%)$ underwent postoperative dialysis in the perioperative period. The incidence of this early postoperative dialysis varied among the hospitals (POWH, 9.1\%; RPAH, 28.6\%; WH, 17.8\%), possibly reflecting the different indications for initiating dialysis among the hospitals. This also varied among the patients within the same hospital and among the intensivists and nephrologists. The specific indications were not analyzed owing to a lack of data; however, the common indications for the initiation of dialysis were refractory fluid overload, hyperkalemia, and acidosis. Multiple logistic regression analysis identified emergency surgery $(\mathrm{OR}, 2.7 ; 95 \% \mathrm{CI}, 1.2-6.2 ; P=.021)$, tricuspid valve surgery (OR, $4.4 ; 95 \% \mathrm{CI}, 1.4-13.3 ; P=.003)$, eGFR $<20 \mathrm{~mL} / \mathrm{min}$ versus $>25 \mathrm{~mL} / \mathrm{min}$ (OR, 3.8; $95 \%$ CI, $1.7-8.4 ; P=.001$ ), and the hospital of surgery as independent predictors of early postoperative dialysis. In the serum creatinine analysis, the same independent predictors were confirmed, along with a serum creatinine level $>350 \mu \mathrm{mol} / \mathrm{L}$ versus 200 to $250 \mu \mathrm{mol} / \mathrm{L}$ (OR, 7.0; $95 \%$ CI, 3.0-16; $P<.001)$. Patient age, bypass time, and the severity of preoperative symptoms were not found to be significant independent risk factors.

\section{New Permanent Dialysis}

Of the 292 preoperative NDD patients who survived the perioperative period, 20 started permanent dialysis within 6 months of surgery. Multiple logistic regression analysis using eGFR and then serum creatinine in separate analyses identified eGFR $<20 \mathrm{~mL} / \mathrm{min}$ (OR, 4.6; 95\% CI, 1.2-17; $P=.024)$ as the only predictor for starting permanent dialysis within 6 months of surgery in the first analysis and preoperative creatinine $>350 \mu \mathrm{mol} / \mathrm{L}$ (OR, 11.6; $95 \%$ CI, 2.2-4.2; $P<.001)$ as the only predictor in the second analysis. Figure 2 illustrates the cumulative risk of death or beginning new permanent dialysis within 6 months

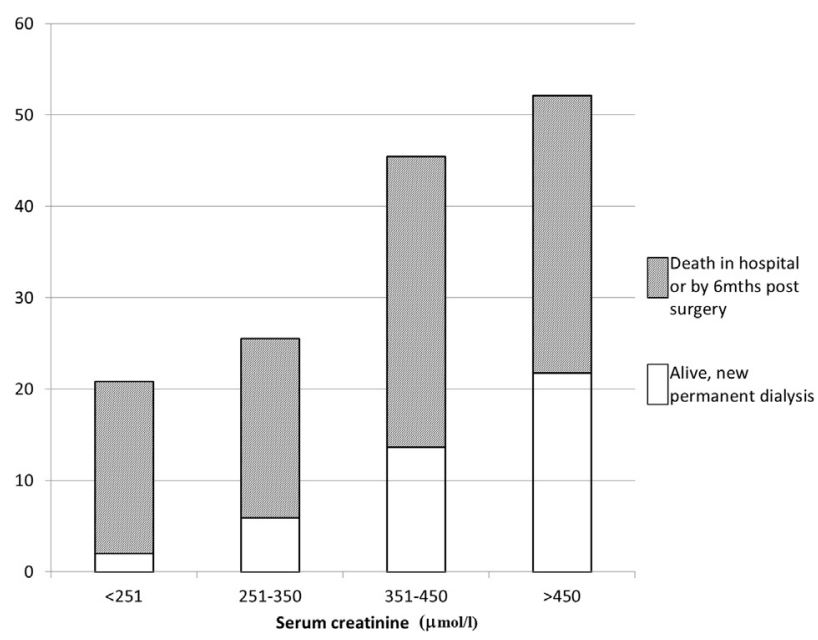

FIGURE 2. Incidence of death or beginning new permanent dialysis within 6 months of surgery according to preoperative serum creatinine level for the 349 preoperative nondialysis-dependent patients.

of surgery by preoperative creatinine level for all 349 NDD patients.

\section{Postdischarge Mortality Within 6 Months of Surgery}

Of the 292 preoperative NDD and 177 DD patients surviving the perioperative period, $16(5.5 \%)$ and $12(6.8 \%)$ died within 6 months of surgery, respectively $(P=.565)$. The independent predictors of death after discharge and within 6 months of surgery were age (OR, 1.6 per decade; 95\% CI, 1.1-2.3; $P=.012)$ and diabetes (OR, 0.25; 95\% CI, 0.09-0.74; $P=.012)$.

\section{Interval to Death for Those Surviving 6 Months Postoperatively ("Late Death Landmark Analysis")}

At the 6-month postoperative landmark, 257 preoperative NDD patients were alive and remained NDD, 19 of the 20 preoperative NDD patients who had become newly DD in the preceding 6 months were alive, and 165 of the preoperative DD patients remained alive and DD. Thus, the overall survival at 6 months was $81 \%$. Multiple Cox regression analysis identified age per decade (OR, 1.4; 95\% CI, 1.2-1.6; $P<.001$ ), female gender (OR, 1.4; 95\% CI, $1.0-1.9 ; P=.042$ ), and preoperative LVEF $<50 \%$ (OR, 1.4; 95\% CI, 1.0-1.9; $P=.040$ ) as independent predictors of the interval to late death from the 6-month landmark. In patients who survived $>6$ months, surgery for endocarditis was associated with improved survival (OR, 0.40; 95\% CI, 0.21-0.78; $P=.006$ ). For patients alive 6 months after surgery, no significant difference was found in the long-term survival between those who had undergone isolated CAG and those who had undergone valve replacement and whether they were DD or NDD at 6 months postoperatively (Figure 3). 

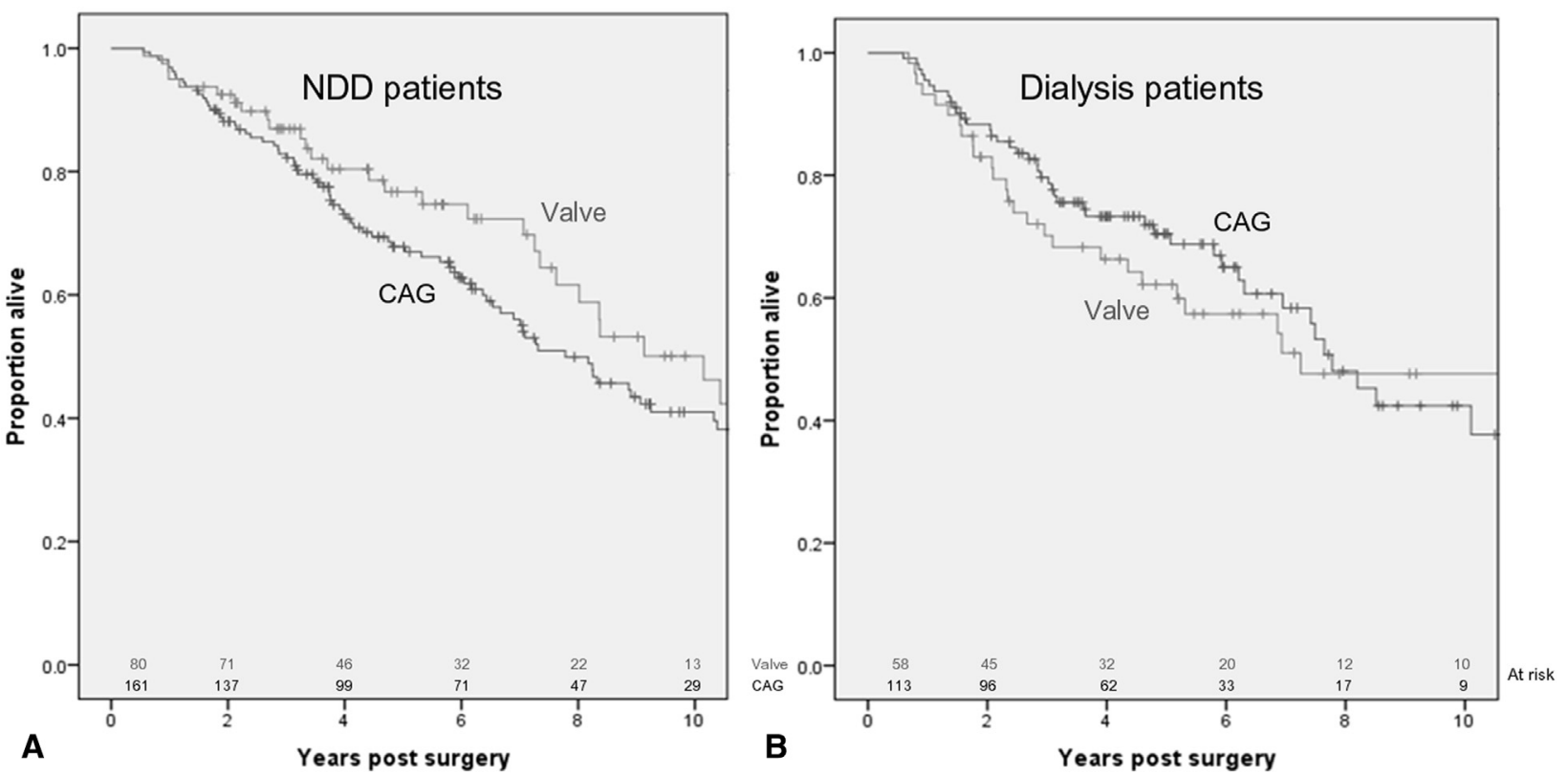

FIGURE 3. The overall survival at 6 months after surgery was $81 \%$ and the Kaplan-Meier survival curves from that point for patients who had undergone isolated coronary artery graft surgery $(C A G)$ and for those who had undergone any valve replacement (Valve) with or without CAG. A, The survival distributions for patients requiring permanent dialysis, with no significant difference between the 2 groups $(P=.781$, log-rank Mantel Cox $)$. B, The survival distributions for patients who did not require permanent dialysis within 6 months after surgery $(P=.801, \log$-rank Mantel Cox $)$. NDD, Nondialysis dependent.

\section{DISCUSSION}

The present multicenter study showed an overall perioperative mortality of $13.9 \%$ and was similar in all 3 units. Several small studies have reported perioperative mortality rates of $11.8 \%$ to $17.1 \% .^{3,4,6-8}$ In an analysis of 483,914 isolated CAG patients from the Society of Thoracic Surgeons database, Cooper and colleagues ${ }^{9}$ reported that the operative mortality increased as the GFR decreased, varying from $1.3 \%$ for a GFR $>90 \mathrm{~mL} / \mathrm{min}$ to $9.3 \%$ for severe renal dysfunction and to $9 \%$ for DD patients. The latter 2 numbers appear more comparable to our study population, because our patients had severe renal dysfunction, with a creatinine of $>200 \mu \mathrm{mol} / \mathrm{L}$. In our study population, the perioperative mortality for isolated CAG was $9.4 \%$, consistent with the $9.3 \%$ reported by Cooper and colleagues. ${ }^{9}$ They also found that preoperative creatinine was the most powerful predictor of mortality after adjustment for other covariants. They also performed analyses including the eGFR using the Modification of Diet in Renal Disease formula and found similar results to the analyses performed using serum creatinine. ${ }^{9}$ Several other investigators have also reported that the perioperative mortality for CAG increased progressively with the preoperative CKD stage. ${ }^{7,10,11}$ This was also true for valve surgery. ${ }^{12}$ Our findings could be representative of Australia, because another group based in Brisbane, Australia, found similar 30-day mortality for 45 chronic dialysis patients who had undergone cardiac surgery. ${ }^{13}$
In our study, the incidence of early postoperative dialysis varied among the 3 hospitals. This might have reflected protocol differences in the thresholds for the use of dialysis in the postoperative period. Early postoperative dialysis was included as a variable for the prediction of the outcomes of perioperative mortality, new permanent dialysis among those discharged, and "late death" in the 6-month landmark analysis. Neither the hospital nor the use of early postoperative dialysis was a significant predictor of any of these outcomes, suggesting that the differing protocols among the hospitals were similarly effective. Others have also described high serum creatinine and emergency surgery as factors that increased the need for postoperative dialysis. ${ }^{4,6}$ Tricuspid valve surgery was an independent predictor of the use of early postoperative dialysis and is likely to be associated with the elevated venous pressure that occurs to varying extents both before and after tricuspid valve surgery. A recent study from the Mayo Clinic reported a $30 \%$ incidence of acute kidney injury after tricuspid valve surgery. ${ }^{14}$

Our data have shown that for the preoperative NDD patients discharged alive, an eGFR $<20 \mathrm{~mL} / \mathrm{min}$ or a preoperative creatinine of $>350 \mu \mathrm{mol} / \mathrm{L}$ was associated with requiring long-term dialysis within 6 months of surgery. This is a question most clinicians face when educating patients about the risks associated with surgery. Although several studies have identified risk factors for requiring postoperative dialysis, few have followed up these 
patients to determine whether they had no longer required dialysis in the long term. ${ }^{4,6,15,16}$

Our study showed that patients with advanced kidney disease who underwent cardiac surgery have 1-, 5-, and 10-year survival rate of $78 \%$ ( $95 \%$ CI, $74 \%-81 \%), 56 \%$ (95\% CI, 52\%-61\%), and 36\% (95\% CI, 32\%-42\%), respectively (Figure 1). However, $65 \%$ of the cohort were considered to require urgent or emergency surgery and faced a high probability of short- or midterm mortality without surgery. In a study of 152 dialysis patients with a similar duration to ours, Takami and colleagues ${ }^{17}$ reported very similar 1-, 5-, and 10-year survival. Our study population included patients with advanced CKD and those requiring dialysis; however, the survival data for DD versus NDD patients were comparable. This finding was similar to that from Thourani and colleagues ${ }^{12}$ in their analysis of 2408 patients undergoing aortic valve replacement. They found that although the long-term survival decreased with each unit decrease in preoperative GFR, the patients with severe renal dysfunction had 10-year survival approximately the same as patients requiring preoperative dialysis. ${ }^{12}$ In a study of 159 patients with renal dysfunction who underwent cardiac surgery, de Peppo and colleagues ${ }^{3}$ also found that the 36 patients with preoperative creatinine $>2.5 \mathrm{mg} / \mathrm{dL}(220 \mu \mathrm{mol} / \mathrm{L})$ had similar long-term mortality to the patients requiring 24 chronic dialysis. In a study comparing the survival of 40,374 patients who underwent cardiac catheterization with that of 662 DD patients and 750 NDD patients with kidney disease who had undergone the same, Hemmelgarn and colleagues ${ }^{18}$ also found that the adjusted 8-year survival rates for CAG were similar at $45.9 \%$ in the NDD kidney disease group and $44.8 \%$ in the DD group. The latter findings were in contrast to those from a study by Chikwe and colleagues. ${ }^{19}$ In their analysis of 2960 patients undergoing isolated CAG, the long-term survival was significantly less for the 96 preoperatively DD patients compared with that of the 61 NDD patients with a preoperative creatinine $>2.5 \mathrm{mg} / \mathrm{dL}$. However, the study by Chikwe and colleagues ${ }^{19}$ only included isolated coronary artery bypass grafting and the others had included valve and other cardiac surgery. Thus, selection bias might have had an effect in the survival of the dialysis patients, because doubts regarding surgical viability could have excluded some patients. Takami and colleagues ${ }^{17}$ identified age $>63$ years, diabetes, and peripheral arterial disease as predictors of late death. Our data identified age and a low LVEF as predictors of the interval to death in those who survived 6 months after surgery. We used serum creatinine $\geq 200 \mu \mathrm{mol} / \mathrm{L}$, rather than the eGFR, to screen for CKD, because this was the parameter recorded in all 3 cardiac surgical databases. Because of their lower muscle mass, the actual renal function might be less in women than in men with an identical creatinine level. In a detailed analysis of the effect of gender on perioperative mortality,
Deutsch and colleagues ${ }^{20}$ found no significant genderspecific difference in 30-day mortality after cardiac surgery. A recent meta-analysis found that although eGFR has been regarded as a better indicator of renal function, it was not superior to creatinine in predicting 30-day mortality when considered as a binary variable. ${ }^{21}$

No difference was found in late survival between those who had undergone isolated CAG and those who had undergone valve replacement surgery with or without other procedures. This was also true in the 2 subgroups that had required permanent late dialysis. No particular type of surgery was associated with worse late survival. Few data are available comparing long-term survival with the surgery type. Our data concur with those from a study of cardiac surgery in 245 DD patients by Rahmanian and colleagues ${ }^{22}$ that reported no difference in late survival when the patients were stratified by the underlying procedure. It is likely the powerful effect of CKD on late survival is such that it largely overshadows the effect of the preoperative cardiac status and the condition requiring treatment. The only cardiac parameter that proved significant was impaired LVEF, and it seems that this, combined with CKD, substantially increases the short- and long-term hazards of surgery.

\section{Study Limitations}

The present study had the inherent limitations of an observational study. Follow-up was through regional databases rather than direct patient contact, and this might have underestimated the late event rate. We used the eGFR without accounting for the body mass as the measure of renal function, and this might have overestimated the renal function in patient groups with a low muscle mass such as the elderly and women. However, although the use of the last serum creatinine level before surgery might have underestimated the stable renal function in some patients with transient worsening, it can indicate the renal function at surgery. Statistical analysis could not completely account for the subtle bias in the selection of these high-risk patients for surgery. The point of cardiac catheterization was not included in the present analysis; therefore, any renal insult from the dye was not analyzed.

\section{CONCLUSIONS}

The results of our multicenter study add to the increasing body of data on the outcomes of cardiac surgery in patients with kidney disease. Although a wide variation was present in the reported perioperative mortality in this group of patients, our data are concordant with those from most studies. A preoperative eGFR of $<20 \mathrm{~mL} / \mathrm{min}$ was associated with starting permanent dialysis after cardiac surgery. The 10-year survival after cardiac surgery has been approximately $35 \%$ to $40 \%$, and this remains true for preoperatively DD and NDD patients and for patients 
undergoing CAG or valve replacement surgery. Our results should assist in providing clinicians and patients with information regarding the implications of cardiac surgery in the presence of CKD.

We thank the doctors, nursing staff, and data managers of the departments of cardiothoracic surgery and nephrology of POWH, RPAH, and WH for their support with data collection.

\section{References}

1. Sarnak MJ. Kidney disease as a risk factor for development of cardiovascular disease: a statement from the American Heart Association Councils on Kidney in Cardiovascular Disease, High Blood Pressure Research, Clinical Cardiology, and Epidemiology and Prevention. Circulation. 2003;108:2154-69.

2. Tonelli M, Wiebe N, Culleton B, House A, Rabbat C, Fok M, et al. Chronic kidney disease and mortality risk: a systematic review. J Am Soc Nephrol. 2006;17:2034-47.

3. de Peppo AP, Parlo N, de Ruggero P, Antonio P, Stefano F, Luigi C. Cardiac surgery in moderate to end-stage renal failure: analysis of risk factors. Ann Thorac Surg. 2002;74:378-83.

4. Durmaz I, Buket S, Atay Y, Yagdi T, Ozbaran M, Boga M, et al. Cardiac surgery with cardiopulmonary bypass in patients with chronic renal failure. J Thorac Cardiovasc Surg. 1999;118:306-15.

5. Mayorchak Y, Paterson HS, Ryan JB, Byth K, Robinson LM, Kovoor P, et al. Mammary artery to saphenous vein composite $\mathrm{T}$ grafts for coronary artery bypass: late follow-up. J Cardiovasc Surg. 2013;54:531-6.

6. Weerasinghe A, Hornick P, Smith P, Taylor K, Ratnatunga C. Coronary artery bypass grafting in non-dialysis-dependent mild-to-moderate renal dysfunction. J Thorac Cardiovasc Surg. 2001;121:1083-9.

7. Bechtel JF, Detter C, Fischlein T, Krabatsch T, Osswald BR, Rieß F, et al. Cardiac surgery in patients on dialysis: decreased 30-day mortality, unchanged overall survival. Ann Thorac Surg. 2008;85:147-53.

8. Yamamura M, Mitsuno M, Tanaka H, Kobayashi Y, Ryomoto M, Nishi H, et al. Risk factors for open heart surgery in hemodialysis patients. Gen Thorac Cardiovasc Surg. 2009;57:235-8.

9. Cooper WA, O'Brien SM, Thourani VH, Guyton RA, Bridges CR, Szczech LA, et al. Impact of renal dysfunction on outcomes of coronary artery bypass surgery: results from the Society of Thoracic Surgeons national adult cardiac database. Circulation. 2006;113:1063-70.
10. Yeo KK, Li Z, Yeun JY, Amsterdam E. Severity of chronic kidney disease as a risk factor for operative mortality in nonemergent patients in the California Coronary Artery Bypass Graft Surgery Outcomes Reporting Program. Am J Cardiol. 2008;101:1269-74.

11. Kinoshita T, Asai T, Murakami Y, Suzuki T, Kambara A, Matsubayashi K. Preoperative renal dysfunction and mortality after off-pump coronary artery bypass grafting in Japanese. Circ J. 2010;74:1866-72.

12. Thourani VH, Keeling WB, Sarin EL, Guyton RA, Kilgo PD, Dara AM, et al. Impact of preoperative renal dysfunction on long-term survival for patient undergoing aortic valve replacement. Ann Thorac Surg. 2011;91:1798-807.

13. Jayasekera H, Pinto N, Mundy J, Wood A, Beller E, Griffin R, et al. Cardiac surgery in the presence of dialysis: effect on mid-term outcomes and quality of life. Heart Lung Circ. 2011;20:105-10.

14. Englberger L, Suri RM, Connolly HM, Li Z, Abel MD, Greason KL, et al Increased risk of acute kidney injury in patients undergoing tricuspid valve surgery. Eur J Cardiothorac Surg. 2013;43:993-9.

15. Hobson CE, Yavas S, Segal MS, Schold JD, Tribble CG, Layon AJ, et al Acute kidney injury is associated with increased long-term mortality after cardiothoracic surgery. Circulation. 2009;119:2444-53.

16. Seabra FV, Alobaidi S, Balk EM, Poon AH, Jaber BL. Off-pump coronary artery bypass surgery and acute kidney injury: a meta-analysis of randomized controlled trials. Clin J Am Soc Nephrol. 2010;5:1734-44.

17. Takami Y, Tajima K, Kato W, Fujii K, Hibino M, Munakata H, et al Predictors for early and late outcomes after coronary artery bypass grafting in hemodialysis patients. Ann Thorac Surg. 2012;94:1940-5.

18. Hemmelgarn BR, Southern D, Culleton BF, Mitchell LB, Knudtson ML, Ghali WA. Survival after coronary revascularization among patients with kidney disease. Circulation. 2004;110:1890-5.

19. Chikwe J, Castillo JG, Rahmanian PB, Akujuo A, Adams DH, Filsoufi F. The impact of moderate-to-end-stage renal failure on outcomes after coronary artery bypass graft surgery. J Cardiothorac Vasc Anesth. 2010;24:574-9.

20. Deutsch O, Spiliopoulos K, Kiask T, Katsari E, Rippinger N, Eichinger W, et al Cardiac surgery in dialysis dependant patients: impact of gender on early outcome in single-center experience with 204 consecutive cases. Thorac Cardiovasc Surg. 2013;61:22-8.

21. Mooney JF, Ranasinghe I, Chow CK, Perkovic V, Barzi F, Zoungas S, et al Preoperative estimates of glomerular filtration rate as predictors of outcomes after surgery. Anesthesiology. 2013;118:809-24.

22. Rahmanian PB, Adams DH, Castillo JG, Vasalotti J, Filsufu F. Early and late outcome of cardiac surgery in dialysis dependent patients: single-center experience with 245 consecutive patients. J Thorac Cardiovasc Surg. 2008; 135:915-21 